\title{
Investigation of Wave Propagation Using Smart Antenna for Indoor Wireless Communication
}

\author{
Junwei Lu and Zhaohui Sun \\ Centre for Wireless Monitoring and Applications, Griffith University, \\ Brisbane, QLD 4111, Australia \\ j.lu@griffith.edu.au and zhaohui.sun@student.griffith.edu.au
}

\begin{abstract}
The paper presents the investigation of wave propagation characteristics for indoor wireless communication using smart mobile terminal antenna. The Finite Element Method (FEM) frequency domain based computation model is used to characterize electric field intensity for a typical office environment. The signal to noise ratio and signal strength are measured according to different placements of transmitting antennas including conventional antenna and smart mobile terminal antenna array. To validate the numerical computation result, measurement result using a wireless network card with an on board antenna and signal strength measurement software are employed to replace expensive and heavy apparatus.
\end{abstract}

\section{INTRODUCTION}

Wireless local area networks (WLANs) have become not only increasingly popular but inevitable. Except for its advantages of freedom, simplicity and ease of use, the security problem in WLAN is more serious than in traditional wired networks. Current solutions are not as successful as expected due to WLAN's inherent specialty of space signal sharing. When the smart mobile terminal antenna is used in WLANs, space can be divided into signal area and non-signal area, or secure area and non-secure area, and thus the system performance can be further improved. In order to satisfy wireless security and EMC/EMI requirements for indoor radio environments, accurate wave propagation characteristics are needed for signal coverage, optimal site design, calculation of system capacity, and other considerations of wireless network installation.

A number of popular numerical simulation methods have been used extensively to perform indoor wireless studies, such as ray-tracing, geometrical optics (GO), uniform theory of diffraction (UTD) [1]-[8], and hybrid ray-tracing-FDTD techniques for similar purposes [9]. Each modeling technique is limited to particular types of models as each numerical technique has its own strengths, and weaknesses. The limitations of practical numerical techniques vary from technique to technique and on the level of detail required in the constructed model. The type of numerical technique chosen depends on the application problem. In this paper, we mainly focus on the measurement of signal strength and the distribution of electric field intensity when a smart mobile terminal antenna array is used as a transmitter. A full wave Finite Element Method (FEM) in frequency domain is employed to solve wave propagation problem for complex indoor radio environment and dielectric embedded antenna array. To enable compatibility with contemporary wireless systems, $2.44 \mathrm{GHz}$ is chosen as the frequency with wavelength in air equal to $\lambda_{0}=12.3 \mathrm{~cm}$.

\section{FEM FREQUENCY DOMAIN APPROACH}

To obtain a full solution to Maxwell's equations for a structure of arbitrary size, a full-wave technique is required. A full-wave computational technique provides a complete solution to Maxwell's equations within the computational space for all conductors and materials. Full wave techniques are more complex than quasi-static techniques, but they are also generic in nature and have fewer limitations in their use [10]. Each full-wave modeling technique is limited to particular types of models. Frequency-domain codes solve for one frequency at a time. This is usually adequate for investigating wave propagation in WLANs with smart mobile terminal antenna used in such a radio environment and for examining specific issues, such as wireless security. Frequency-domain approaches are in general faster than their time-domain approaches. Therefore, several frequencydomain simulations can usually be run in the time it would take for a single time-domain simulation. A further benefit to using frequency-domain codes is their capacity to use larger meshes for the lower frequencies, which in turn permits a shorter computation time. To cover a wide frequency range with frequency-domain codes, a number of simulations are required. In this simulation we only investigate the $2.4 \mathrm{GHz}$ frequency range which is used for IEEE wireless communication systems.

\section{A. Numerical Model for Radio Environment}

The simulation model for a typical office area is illustrated in Fig. 1, where three potential placements of the transmitting antenna are indicated. The FEM based numerical simulator FEMLAB [11] is employed to solve the electric field distribution where the frequency domain vector wave equation for $\boldsymbol{E}$ field can be derived as:

$$
\nabla \times \frac{1}{\mu} \nabla \times E+\omega^{2} \varepsilon E=-j \omega J
$$

where $\omega$ is angular frequency, $\boldsymbol{J}$ is source current, $\sigma_{\mathrm{e}}$ is the effective conductivity, $\mu$ and $\varepsilon$ are the permeability and permittivity of the problem space respectively. 


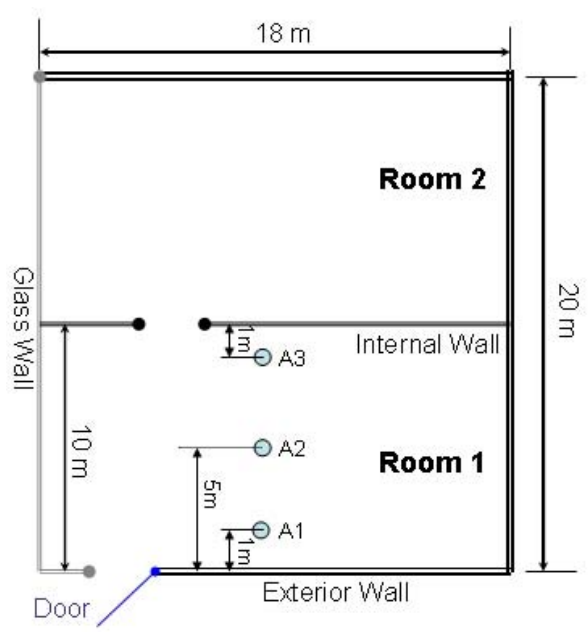

Fig. 1. Layout of the examined office area where three potential placements of the transmitting antenna are indicated.

As depicted in Fig. 1, the indoor experiment environment is a typical office area including two separate rooms. The dimensions of the examined region are $20 \mathrm{~m} \times 18 \mathrm{~m}$, three exterior walls are made of homogeneous $20 \mathrm{~cm}$ brick block with conductivity equal to $\sigma=4.7 \mathrm{mS} / \mathrm{m}$ and a relative dielectric constant of $\varepsilon_{\gamma}=7$, and the fourth is glass windows, placed at the left-hand side of rooms 1 and 2, while we have $\sigma=\mathrm{e}^{-14} \mathrm{~S} / \mathrm{m}$ and $\varepsilon_{\gamma}=4.2$. Rooms 1 and 2 are separated by a thinner $(10 \mathrm{~cm})$ partition, mainly made of plastic, whose parameters are $\sigma=0 \mathrm{~S} / \mathrm{m}$ and $\varepsilon_{\gamma}=3$. We give the air in the room $\sigma=0 \mathrm{~S} / \mathrm{m}$ and $\varepsilon_{\gamma}=1$ and all the materials $\mu_{\gamma}=1$.

\section{B. Antenna Model used in Simulation}

Using a directional antenna in wireless Accesses Point (AP) or terminal, the security level can be greatly improved in WLAN, while the influence in AP's case is more distinct. The narrower the bandwidth, the better performance will be achieved for a certain wireless communication environment. In this simulation a 13-element dielectric-embedded electronically switched multiple-beam (DE-ESMB) antenna array [12] is adopted to form a single fixed-beam directional antenna. As shown in Fig. 2 (a)-(b), this antenna consists of one center grounded element and two radially concentriccircle surrounding elements where elements in the inner circle are active elements, the other elements in the outer circle are passive elements. The parameters of copper material for antenna elements with the conductivity $\sigma=5.99 \mathrm{e}^{7} \mathrm{~S} / \mathrm{m}$ and permittivity $\varepsilon_{\gamma}=1$; and the embedded dielectric material with the conductivity parameters $\sigma=\mathrm{e}^{-14} \mathrm{~S} / \mathrm{m}$ and $\mathcal{E}_{\gamma}=4.2$ are used in the FEM frequency domain simulation.

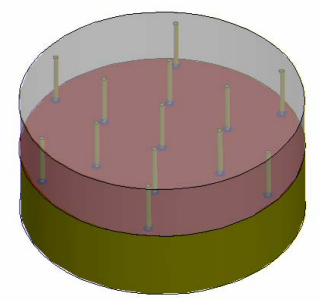

(a) DE-ESMB antenna

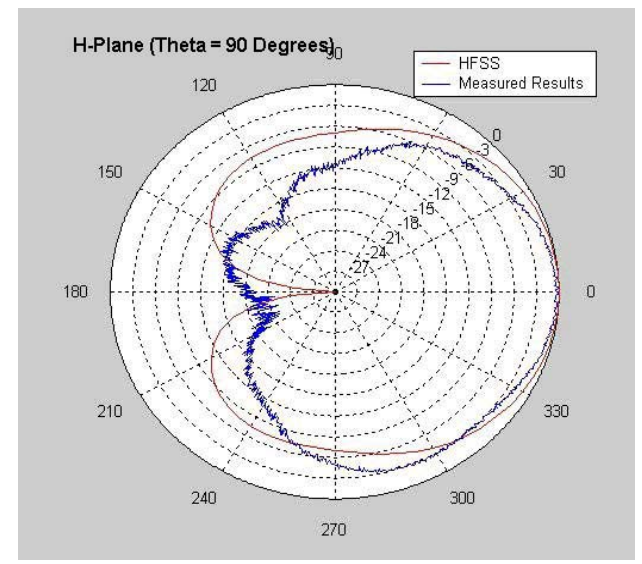

(b) Switched directional beam

Fig. 2. Top view of 13-element DE-ESMB antenna and its radiation pattern.

\section{SIMULATION RESULTS}

Figure 3 shows the FEM mesh for the simulation domain where three potential positions of the transmitter are examined. For the reduction of undesirable reflections from the domain's boundaries, a low-reflecting layer is used. All simulations consider the case of TE wave and time-harmonic variations are assigned to the transmitter's position according to the operation frequency. The mesh resolution is assigned at 23048 elements. The channel behavior can be investigated with respect to the relative source positioning.

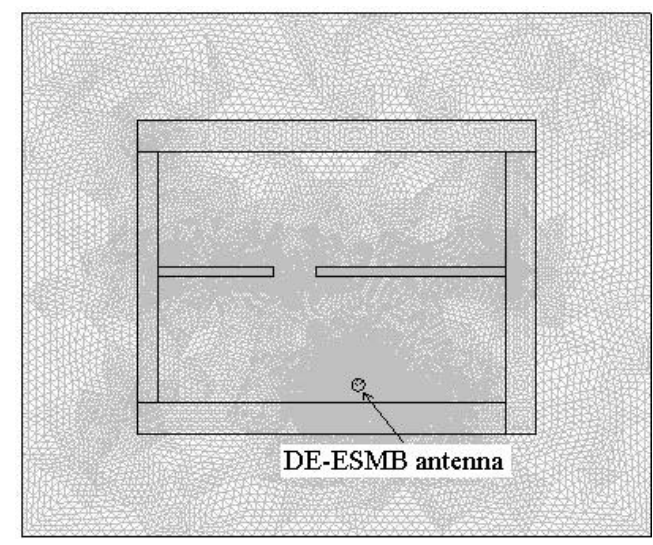

Fig. 3. FEM mesh of the simulation domain including indoor and part of outdoor with DE-ESMB antenna located at A1 position. 
Figure 4 presents simulation results of the electric field intensity distribution using omini-directional antenna as a transmitter located at position A1. The simulation result using a fixed directional DE-ESMB antenna as transmission source in different positions are shown in Figure 5 and 6. The signal leaking behind the source and outside of the room is sporadic compared with omni-directional case. This is because, although the back direction does not contain the main lobe of the antenna, the reflected and diffracted wave still can penetrate the wall, but the signal strength already gets weak. This does reveal the predominant potential of using directional antenna in WLAN to improve the security performance and satisfy EMC/EMI requirement in particular wireless application environment.
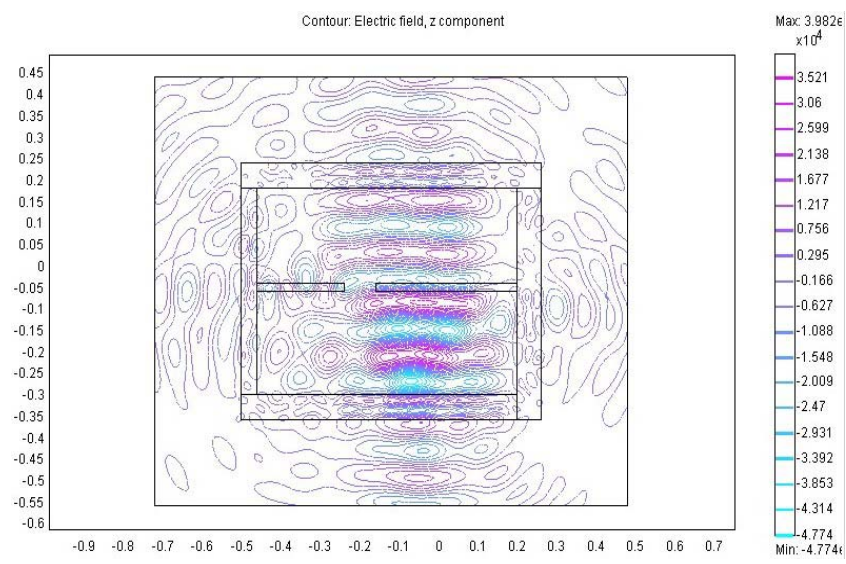

Fig. 4. 2D electric field intensity distribution using omni-directional antenna as a transmitter located at position A1.

Contour. Electric field, z component
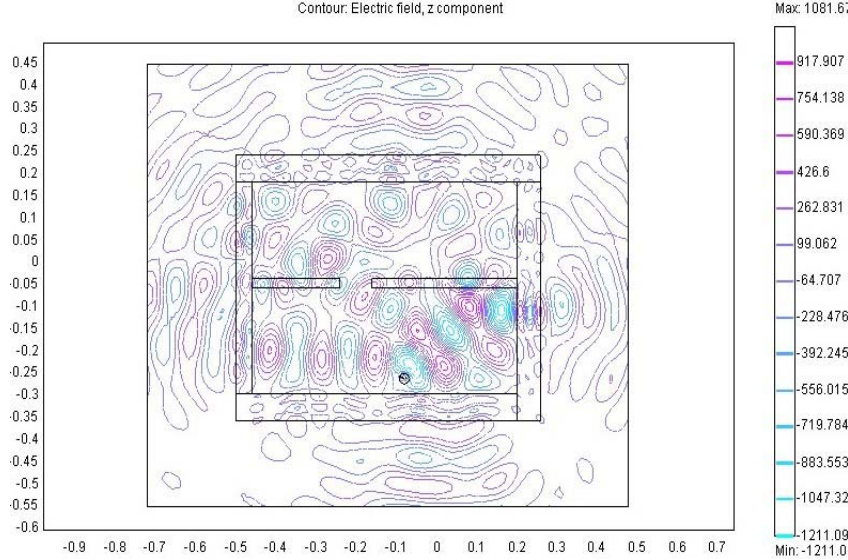

(a) DE-ESMB antenna as a transmitter located at position A1

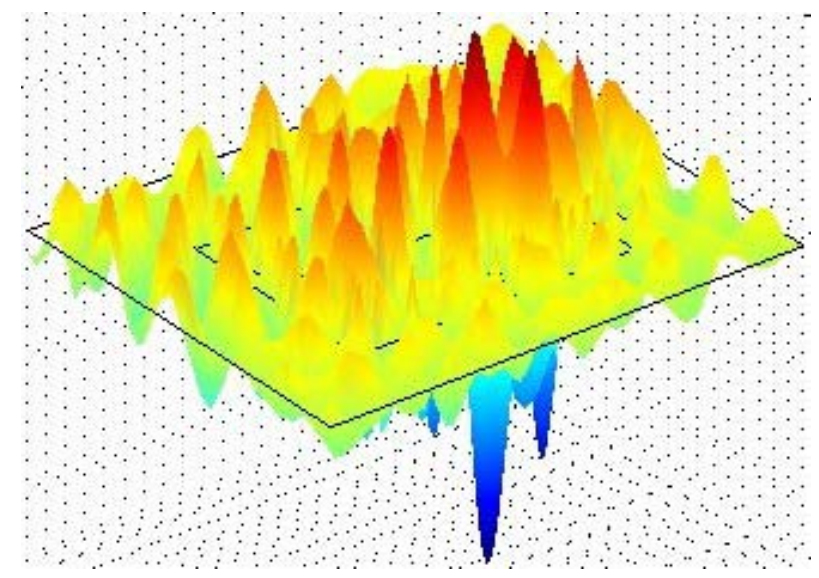

(b) 3D plot of the electric field intensity distribution in

Fig.5. Electric field intensity distribution using DE-ESMB smart antenna located at position $\mathrm{A} 1$.

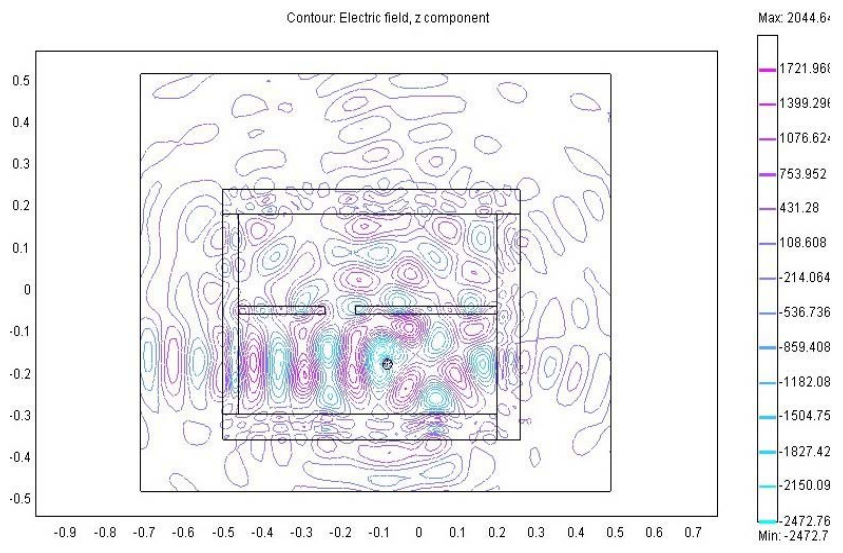

(a) DE-ESMB antenna as a transmitter located at position A2

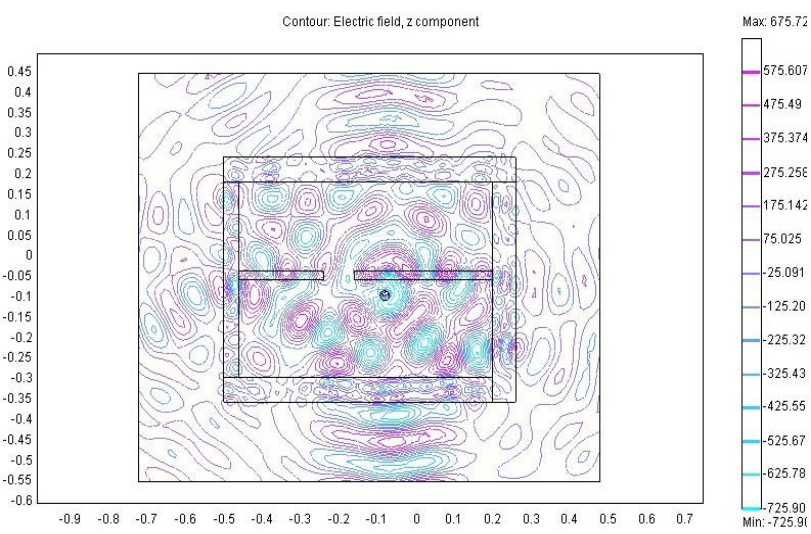

(b) ) DE-ESMB antenna as a transmitter located at position A3

Fig. 6. 2D electric field intensity distribution map using DE-ESMB smart antenna as a transmitter located in two different positions.

By taking into account the results from all transmitter positions, some interesting observation can be made. Due to shadowing effect of the thin wall, the mean electric field 
intensity in Room 1 is always stronger than in Room 2. In addition, within Room 1, the left side of the source is slightly weaker than the right side. This finding can be explained by the fact that the glass walls have a smaller relative permittivity ( $\varepsilon_{\gamma}=4.2$ ) than brick wall $\left(\varepsilon_{\gamma}=7\right.$ ) and comparatively shorter width (1 cm and $20 \mathrm{~cm}$, respectively), it causes less reflection and diffraction, more energy from the transmitter can propagate outside the room than that for the brick wall cases.

\section{EXPERIMENT RESULTS}

To examine the smart antenna performance in a real environment, a typical office area consisting of two rooms was used, where the layout of the environment is shown in Fig 7. The dimensions of the examined region are $20 \times 18 \mathrm{~m}$, where three walls are made of $40 \mathrm{~cm}$ brick block and the fourth is a large glass window. Six different positions are chosen to compare the signal strength. The following wireless equipments were adopted: (a) Accesses Point: D Link DWL 900AP+ (Firmware: rev. B 2.61), (b) 2) Terminal: COMPAQ PII notebook with D_Link DWL_660 802.11b wireless card.

In order to enable comparability of the result, six different antennas are used, such as two D_Link equipment embedded antennas; two benchmark antennas come from M.gear Co., and two smart antennas, named ESPAR [14] and DE-ESMB antenna arrays [12]. Network Stumbler 0.4.0 software [15] is also employed to measure the signal-to-noise ratio and signal strengths. From the experiment results, one can see that using MGR-DS-06B in AP, ESPAR in terminal; and ESMB in AP, ESPAR in terminal, whose corresponding security level was significantly increased [13].

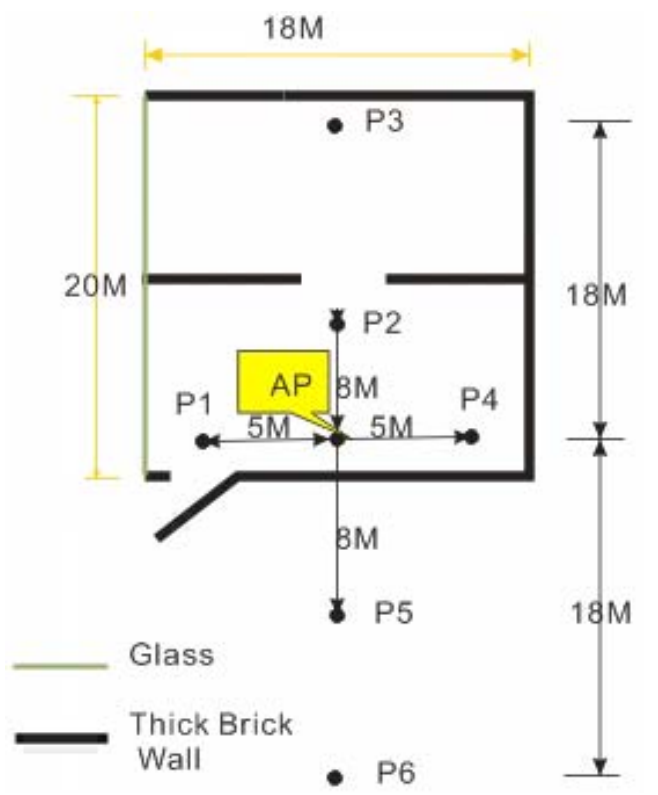

Fig. 7. Measurement of signal strength using Smart antenna located in AP.
TABLE I

INDOOR EXPERIMENT RESULTS USING DELL LATITUDE D-400 LAPTOP COMPUTER WITH EMBEDDED ANTENNA

\begin{tabular}{|c|c|c|c|c|c|c|}
\hline \multirow{2}{*}{ Transmit Antenna } & \multicolumn{5}{|c|}{ Signal/Noise Ratio (dBm) } \\
\cline { 2 - 7 } & P1 & P2 & P3 & P4 & P5 & P6 \\
\hline $\begin{array}{c}\text { D_Link DWL_900AP } \\
\text { omni-directional }\end{array}$ & -28 & -36 & -55 & -30 & -52 & -55 \\
\hline $\begin{array}{c}\text { M.gear 6dBi } \\
\text { directional }\end{array}$ & -28 & -30 & -50 & -28 & -58 & -58 \\
\hline $\begin{array}{c}\text { DE-ESMB } \\
\text { directional }\end{array}$ & -32 & -38 & -52 & -33 & -62 & -63 \\
\hline
\end{tabular}

Table I illustrates signal/noise ratio with three different antennas used at AP position in the WLAN. It is evident that the average power outdoors is weaker with the AP using directional antenna (beam front facing P3 direction) in Position A1, which indicates that the optimal position of AP can effectively decrease the unwanted signal leaking, thus greatly improving the security level. It is also worth noticing that using the same gain and transmit power, the smart antenna exhibited better performance than the commercial M.gear directional antenna. Such advantage results from its comparatively narrow beamwidth since it can concentrate the electromagnetic energy on given direction.

While the level of Signal to Noise ratio at these positions is relatively low, the electric field intensity from simulation result at observation position $\mathrm{P} 5$ and $\mathrm{P} 6$ is quite smaller when DE-ESMB antenna located at A1 position. Since the performance of wireless communication depends on the electromagnetic characteristics of complex environment, the electric field distribution is dominated by scattering processes due to obstacles, such as wall, furniture, people moving around and so forth. Table II presents the comparison between electric field intensity and Signal to Noise ratio, which can further describe the characteristics of field distribution in wireless communication environment.

\section{TABLE II}

ELECTRIC FIELD INTENSITY AND SIGNAL-TO-NOISE RATIO

\begin{tabular}{|l|l|l|l|l|}
\hline \multirow{2}{*}{ Font Size } & \multicolumn{2}{|c|}{ P5 } & \multicolumn{2}{c|}{ P6 } \\
\cline { 2 - 5 } & $\begin{array}{l}\text { Field } \\
\text { strength } \\
(\mathrm{V} / \mathrm{m})\end{array}$ & $\begin{array}{l}\text { Signal-to- } \\
\text { Noise ratio } \\
(\mathrm{dBm})\end{array}$ & $\begin{array}{l}\text { Field } \\
\text { strength } \\
(\mathrm{V} / \mathrm{m})\end{array}$ & $\begin{array}{l}\text { Signal-to- } \\
\text { Noise ratio } \\
(\mathrm{dBm})\end{array}$ \\
\hline $\begin{array}{l}\text { Omni- } \\
\text { directional }\end{array}$ & $>1.217 \times$ & -52 & $\begin{array}{l}>1.217 \times \\
10^{4}\end{array}$ & -55 \\
\hline DE-ESMB & $<100$ & -62 & $<100$ & -63 \\
\hline
\end{tabular}

\section{CONCLUSIONS}

This paper proposed a model for prediction of electromagnetic environments using directional transmission antenna based on the Finite Element Method. The directionality and security benefit can be clearly observed from the 2D and 3D figures. To validate the numerical results, an experimental method using Network Stumbler software to measure the signal strength in different location was also 
introduced. The comparison of numerical and experimental results showed a satisfactory agreement between the two methods. The latter method gives the users the flexibility of moving around to detect the real-time signal strength in different locations using a laptop instead of expensive measuring apparatus. Such advantage can be very valuable for efficient implementing of indoor wireless networks with security concerns.

\section{REFERENCES}

[1] R. P. Torres, S. Loredo, L. Valle, and M. Domingo, "An accurate and efficient method based on ray-tracing for the prediction of local flatfading statistics in picocell radio channels," IEEE J. Select. Areas commun,. Vol. 19, pp. 170-178, Feb. 2001.

[2] P. Bernardi, R. Cicchetti, and O. Testa, "An accurate UTD model for the analysis of complex indoor radio environments in microwave WLAN systems, "IEEE Trans. Antennas Propagation. Vol. 52, pp. 1509-1520, June 2004

[3] Z. Zhang, R. K. Sorensen, Z. Yun, M. F. Iskander, and J. F. Harvey, "Complex-wall effect on propagation characteristics and MIMO capacities for an indoor wireless communication environment," IEEE Trans. Antennas Propagation, Vol. 52, pp. 914-922, Apr. 2004.

[4] T. Schöberl, "Combined Monte Carlo simulation and ray tracing method of indoor radio propagation channel," in IEEE MTT-S Int. Microwave Symp. Dig., New York, May 16-20, 1995, pp. 1379-1382

[5] M. Kimpe and H. Leib, "Computerized indoor radio channel estimation using ray tracing," Ann. Telecommunications, vol. 52, pp. 251-263, May-June 1997.

[6] S. Chen and S. Jeng, "An SBR/image approach for radio wave propagation in indoor environments with metallic furniture," IEEE Trans. Antennas Propagat., vol. 45, pp. 98-106, Jan. 1997.

[7] C. Chiu and C.Wang, "Impact of shapes of buildings and metallic furniture on indoor multipath environment and bit-error rate performance of millimeter-wave channels," Microwave Opt. Technol. Lett., vol. 19, no.2, pp. 137-142, Oct. 5, 1998.

[8] V. Degli-Esposti, G. Lombardi, C. Passerini, and G. Riva, "Wide-band measurement and ray-tracing simulation of the $1900-\mathrm{MHz}$ indoor propagation channel: Comparison criteria and results," IEEE Trans. Antennas Propagat., vol. 49, pp. 1101-1110, July 2001.

[9] Y. Wang, S. Safavi-Naeini, and S. K. Chaudhuri, "A hybrid technique based on combining ray tracing and FDTD methods for site-specific modeling of indoor radio wave propagation," IEEE Trans. Antennas Propagation,. Vol. 48, pp. 743-754, May 2000.

[10] B. Archambeault, C. Brench and O. M. Ramahi, EMI/EMC Computational Modeling Hand Book, Second Edition, Kluwer Academic Publishers, 2001.

[11] FEMLAB v3.1. COMSOL Corp... [Online Available]: http://www.comsol.com.

[12] Junwei Lu, David Thiel, Seppo Saario, "FDTD Analysis of DielectricEmbedded Electronically Switched Multiple Beam Antenna Array," IEEE Trans. Magnetics, vol. 38, pp. 740-741, March 2002.

[13] Zhaohui Sun, Junwei Lu, David Ireland, "Increased Security Level using Space-division Approach in Wireless Computing Network," APMC 2005, Proceedings of APMC-2005, Vol. 3. pp 1979-1982, Dec. 2005.

[14] J. Lu, D. Ireland, and R. Schlub, "Dielectric embedded ESPAR (DEESPAR) antenna array for wireless communications," IEEE Transactions on Antennas and Propagation, vol. 53, no. 8, pp. 24372443, Aug. 2005

[15] Network Stumbler $\quad 0.4 .0, \quad$ [Online Available]:http://www.netstumbler.com. 